\title{
miR-155 is positively regulated by CBX7 in mouse embryonic fibroblasts and colon carcinomas, and targets the KRAS oncogene
}

Floriana Forzati ${ }^{1}$, Marco De Martino ${ }^{1}$, Francesco Esposito ${ }^{1}$, Romina Sepe ${ }^{1}$, Simona Pellecchia', Umberto Malapelle², Gianluca Pellino ${ }^{3}$, Claudio Arra $^{4}$ and Alfredo Fusco ${ }^{1 *}$

\begin{abstract}
Background: Loss of CBX7 expression has been described in several malignant neoplasias, including human colon and thyroid carcinomas proposing CBX7 as a tumor suppressor gene with a key role in cancer progression. This role is supported from the development of benign and malignant neoplasias in Cbx7 null mice.

The aim of our work has been to investigate the mechanisms underlying the CBX7 oncosuppressor activity by analyzing the microRNAs (miRNAs) regulated by CBX7.

Methods: The miRNA expression profiles of the mouse embryonic fibroblasts (MEFs) null for Cbx7 and the wild-type counterpart were analyzed by the miRNACHIP microarray and then validated by qRT-PCR. To asses KRAS as target of miR-155 we evaluated the protein levels after transfection of the synthetic miR-155. Human colon carcinoma samples have been investigated for the expression of CBX7 and miR-155.

Results: Twenty miRNAs were found upregulated and nine, including miR-155, downregulated in cbx7-null MEFS in comparison with the wild-type ones. Then, we focused on miR-155 since several studies have shown its deregulated expression in several human malignancies and, moreover, was the most downregulated miRNA. Subsequently, we searched for miR-155 target genes demonstrating that KRAS protein levels are directly modulated by miR-155. A direct significant correlation ( $r=0.6779$ ) between CBX7 and miR-155 expression levels was found in a set of human colon carcinoma tissue samples.
\end{abstract}

Conclusion: miR-155 is positively regulated by CBX7 in MEFs and colon carcinomas, and has KRAS as one of the target genes likely accounting for the anti-apoptotic activity ascribed to miR-155 in some tissue contexts.

Keywords: CBX7, miR-155, KRAS, Colon carcinoma

\section{Background}

CBX7 belongs to the polycomb repressive complex 1 (PRC1), a multiprotein complex that together with the polycomb repressive complex 2 (PRC2) inhibits the transcription of the developmental genes [1-3]. A crucial activity of $\mathrm{CBX7}$ in tumor progression is supported by

\footnotetext{
* Correspondence: alfusco@unina.it

${ }^{1}$ Istituto di Endocrinologia ed Oncologia Sperimentale "G. Salvatore" - CNR c/o Dipartimento di Medicina Molecolare e Biotecnologie Mediche, Università degli Studi di Napoli "Federico II", Naples, Italy

Full list of author information is available at the end of the article
}

several studies [4]. Indeed, a drastic downregulation of CBX7 expression has been described in thyroid [5], pancreatic [6], colon [7], lung [8], gastric [9], bladder [10], breast [11] carcinomas, and a more advanced stage of neoplastic disease and a poor survival has been directly correlated to the loss of $C B X 7$ expression [6, 7]. Furthermore, when $C B X 7$ expression is restored in thyroid [5], gastric [9] and colon [7] carcinoma cells there is a decreased proliferation rate with the accumulation of the cells in the G1 phase of the cell cycle, suggesting a negative role of CBX7 on the control of cell growth and, 
particularly, in the regulation of the G1/S switch of the cell cycle [5].

CBX7 is able to interact with different proteins, modulating in positive or negative the expression of several genes implicated in various biological functions [4]. In particular, it positively regulates the expression of Ecadherin [12] that is required to maintain the regular morphology of epithelial cell, and whose loss of expression is associated with the epithelial-mesenchymal transition [13, 14]. The CBX7 activation of E-cadherin expression is caused by the interaction with histone deacetylase 2 and inhibition of its action on the CDH1 promoter [12]. Consistently, two studies, in thyroid [12] and pancreatic [6] carcinomas, report a direct correlation between the $C B X 7$ expression and the E-cadherin levels.

Moreover, we have also shown that CBX7 counteracts the HMGA-induced activation of the SPP1 gene [15], encoding the chemokine osteopontin, that is highly overexpressed in several human carcinomas and has a key function in malignant transformation. Furthermore, CBX7 inhibits, by a similar mechanism, the promoter activity of Cyclin E gene [8], that enhances the transition of the G1 to $\mathrm{S}$ phase of the cell cycle then increasing the cell proliferation rate. Therefore, on the basis of all these studies, the absence of the $C B X 7$ gene expression plays an important function in the late stages of human malignancies [4].

MicroRNAs (miRNAs) have become known as a significant class of short endogenous RNAs that control gene expression at post-transcriptional level through base-pairing with their target mRNAs for direct cleavage or by inhibiting mRNA translation [16-18]. They have a central function in a lot of biological pathways, as developmental process, signaling transduction, stem cell differentiation, cell growth, and cancer [19, 20].

In the current study, we have carried out an analysis of miRNA expression profiling in mouse embryonic fibroblasts (MEFs) obtained from Cbx7-knockout (KO) and wild-type (WT) mice to search for the miRNAs modulated by the $\mathrm{Cbx} 7$ protein. Among the differentially expressed miRNAs in WT and Cbx7-null MEFs, we concentrated our attention on the miR-155, downregulated in homozygous $C b x 7-\mathrm{KO} \mathrm{MEFs}$ in comparison to the WT. Consistently, we report downregulation of both $C B X 7$ and miR-155 expression in a set of colon carcinomas. Finally, we demonstrate that KRAS gene is a target of miR-155.

\section{Methods}

\section{Cell cultures}

Primary MEFs from $C b x 7^{+/+}, C b x 7^{+/-}, C b x 7^{-1}$ and transgenic (TG) $C b x 7$ mice, were obtained and grown as previously described [8].
Human embryonic kidney HEK 293 cells and lung cancer cell lines A549 were cultured as reported elsewhere $[8,21]$.

\section{miRNACHIP microarray}

RNA labeling, hybridization on miRNA microarray chips and microarray analyses were carried out as reported elsewhere [22, 23].

Briefly, $5 \mu \mathrm{g}$ of total RNA from each sample were biotin-labeled through reverse transcription by random examers. Hybridization was performed on a miRNA microarray chip [22] containing 368 probes in triplicate. Hybridization signals were identified through biotin binding of a streptavidin-alexa 647 conjugate by a Perkin-Elmer ScanArray XL5K (Perkin-Elmer, Wellesley, MA, USA) and quantified by the Quantarray software (Perkin-Elmer). Raw data were normalized and analyzed by GENESPRING software (Silicon Genetics, Redwood City, CA, USA). miRNAs were quantified by class comparison using Student's $t$ test procedure. Each sample was studied for miRNA expression profile in triplicate.

\section{Bioinformatic prediction of miRNA target genes}

Genes potentially targeted by the selected miRNAs were found through on-line accessible tools i.e. miRanda (www.microrna.org), TargetScan (www.targetscan.org), or miRwalk (http://zmf.umm.uni-heidelberg.de/apps/ $\mathrm{zmf} /$ mirwalk2)

\section{RNA extraction, reverse transcription and quantitative real time (qRT-PCR)}

Total RNA was extracted from cells and tissue samples using Trizol (Invitrogen, Carlsbad, CA), according to manufacturer's instructions. In particular, tissue samples were dissociated with homogenizer. For mRNA detection, $1 \mathrm{mg}$ of RNA from each sample was reversetranscribed using QuantiTech Reverse Transcription kit (Qiagen, Valencia, CA) [24] and then Real-Time PCR was performed by using iQ SybrGreen SuperMix (BioRad, Hercules, CA). qRT-PCR analyses were performed using the following primers:
h CBX7-fw 5'-cgagtatctggtgaagtggaaa-3'
h CBX7 rev 5'-gggggtccaagatgtgct-3'
h KRAS-fw 5'-aggctcaggacttagcaagaa-3'
h KRAS-rev 5'-gaaggcatcatcaacaccct-3'
m KRAS-fw 5' - tgtggatgagtatgaccctacg-3'
m KRAS-rev 5' - ccctcattgcactgtactcct-3'

For miRNA detection, RNA from each sample was reverse-transcribed using miScript reverse transcription Kit (Qiagen, Valencia, CA). Real-Time PCR was performed according to miScript System Kits (Qiagen, Valencia, CA) instructions [24]. Real-Time PCR reactions 
contained miScript Primer Sets (Qiagen, Valencia, CA), specific for mir-155 (5'-UUAAUGCUAAUCGUGAU AGGGGU-3') and U6 (cod. MS00033740) (used to normalize RNA levels).

\section{Plasmids and transfections}

miRNA transfection were performed as described elsewhere [23].

The KRAS 3'-UTR region, containing binding sites for miR-155, was amplified by PCR using the primers KRAS fw 5'-aatttctagaggcatactagtacaagtggt-3' and KRAS rev 5'aatttctagacagggatgacaaactatagg-3'. The amplified fragments were cloned as previously described [23]. Mutation of miR155-binding site (acgtaaa) was synthesized by IDT (TEMA ricerca, Bologna, Italy).

\section{Protein extraction, western blotting, and antibodies}

Protein extraction and Western blotting were performed as previously described [8]. Primary antibodies used were anti-KRAS (H00003845-M01, Abnova and sc-30, Santa Cruz), anti-Actin (sc-1616, Santa Cruz) antiGapdh (sc-32233, Santa Cruz). Western blotting detection reagents (Thermo Scientific, Rockville, IL) were used to visualize immunoblots.

\section{Dual-luciferase reporter assay}

$4 \times 10^{4} 293$ cells were co-transfected in 24-well dishes with the pGL3-KRAS 3'-UTR luciferase reporter construct or with the mutated KRAS 3'-UTR luciferase vector, together with the Renilla luciferase plasmid and with the RNA oligonucleotides (Ambion), using lipofectamine plus (Ambion). The pRL-TK control vector expressing Renilla luciferase (Promega) was used to normalize cell number and transfection efficiency. Luciferase activity was measured as previously reported [25].

\section{Collection and processing of surgical resections}

Carcinoma and normal colon samples were obtained from patients undergoing surgery for colorectal carcinoma (CRC) at two University Hospitals (Second University of Naples and University "Federico II"). Following surgical resection of the bowel tract, a fragment of the tumor and non-pathological tissue were collected from the fresh specimen. Then, samples were divided into several fragments for future extractions of nucleic acids (DNA and RNA) and proteins. Fractionated tissue samples for extractions were then stored at $-80{ }^{\circ} \mathrm{C}$.

\section{Statistical analysis}

Statistical analyses were performed through using GraphPad Prism. The comparison between two groups of experiments was carried out using Student's $t$ test. Results are reported as means $\pm \mathrm{SD}$ and differences were considered to be significant with $p<0.05$.

\section{Results}

MiRNA expression profile of MEFs isolated from Cbx7knockout ( $\mathrm{Cb} \times \mathrm{7} \mathrm{KO}$ ) mice

First, we analyzed by the miRNACHIP microarray [22] the expression profile of miRNAs in MEFs deriving from WT and $C b x 7 \mathrm{KO}$ mice to identify the miRNAs differentially expressed in MEF null for $C b x 7$ in comparison with the WT counterpart. Then, we applied biostatistical analysis (see Methods section), acquiring a list of miRNAs that were differentially expressed $(P<0.05)$ between WT and homozygous Cbx7 KO MEFs (Tables 1 and 2).

The next step was to validate the data acquired by microarray Chip, analyzing 5 deregulated miRNAs by qRT-PCR. The results presented in Fig. 1 confirm a drastic overexpression of miR-181, miR-137, miR-199, miR-706 and miR-719 and repression of miR-155 in Cbx7 KO MEFs in comparison with the WT ones.

Then, we focused on the miR-155, which revealed the most decreased fold-change in Cbx7-null MEFs (Table 1). Moreover, its expression has been frequently found deregulated in human malignancies [26, 27], and its oncogenic activity has been described also in vivo [28]. We then extended the analysis of miR 155 expression, by qRT-PCR, also to MEFs overexpressing $C b x 7$ [8] and other $C b x 7 \mathrm{KO}$ MEF preparations. As presented in the Fig. 2a and b, miR-155 levels decrease in KO MEFs in comparison with the WT ones and it was upregulated in those overexpressing $C b x 7$.

\section{KRAS oncogene is target of miR-155}

To identify possible mRNA targets of the CBX7-regulated miRNAs, we referred to different on-line available bioinformatic tools (see Methods). Among the numerous potential targets of the miR-155, we focused on KRAS because of its key role in development and cancer. Indeed, mutations in the KRAS gene have been recurrently unveiled in numerous neoplasias comprising lung, pancreatic and colon carcinomas [29-31], where we have previously found a significant downregulation of $\mathrm{CBX} 7$ expression. Moreover, it has also been reported an increased KRAS expression in human colon carcinomas [32].

In Fig. 3 Panel a, we show that 3'UTR of KRAS has one putative binding site for the miR-155. Then, to confirm the effect of the miR-155 on the selected candidate target, we evaluated KRAS protein levels in 293 cells transfected with the miR-155, by western blot analysis. As presented in Fig. 3b, introduction of the miR-155 critically decreases the KRAS protein levels. Conversely, no variations in KRAS protein levels were found when the cells were transfected with a scrambled oligonucleotide. Interestingly, modifications in the KRAS mRNA amounts were detected in the cells transfected with the miR-155 (Fig. 3c), thus indicating a role of miR-155 in KRAS mRNA degradation. 


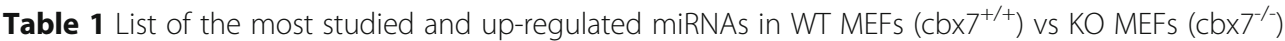

\begin{tabular}{|c|c|c|c|c|}
\hline Unique Id & $\begin{array}{l}\text { Geom. Mean of intensities } \\
\left(\mathrm{cb} \times 7^{+/+}\right)\end{array}$ & $\begin{array}{l}\text { Geom. Mean of intensities } \\
\left(\mathrm{cb} \times 7^{-1-}\right)\end{array}$ & $\begin{array}{l}\text { Ratio of geom } \\
\text { Means } \\
\left(\mathrm{cb} \times 7^{+/+} \mathrm{vs} \mathrm{cb} \times 7^{-/}\right)\end{array}$ & $\begin{array}{l}\text { Parametric } \\
p \text {-value }\end{array}$ \\
\hline Mmu-mir-705 & 626.5 & 205.8 & 3.044 & 0.0062209 \\
\hline Mmu-mir-221 & 4233.9 & 2449.4 & 1.729 & 0.0035147 \\
\hline Mmu-mir-222 & 12079.2 & 6409 & 1.885 & 0.0017689 \\
\hline Mmu-mir-715 & 127.5 & 37.2 & 3.427 & 0.0016821 \\
\hline Mmu-mir-323 & 1775 & 633.6 & 2.801 & 0.0003329 \\
\hline Mmu-mir-155 & 2720.1 & 508.9 & 5.345 & $7.11 \mathrm{e}-05$ \\
\hline
\end{tabular}

Several miRNAs regulate gene expression through base pairing with the miRNA-recognizing elements (miR-RE) in their mRNA target. To establish whether the direct interaction between the miR-155 and KRAS mRNA is responsible for reduced expression of this protein, we introduced downstream of the luciferase ORF, 485 bp (25$510 \mathrm{bp}$ ) of the 3'-UTR of the KRAS mRNA. 293 cells were transfected with the generated reporter vector and the miR-155. Luciferase activity was much lower after miR155 transfection (Fig. 3d) than after transfection with the scrambled oligonucleotide. The same reporter construct of the previous experiments, but carrying mutations in KRAS 3' UTR at the miR-155 target site, was unresponsive to the effects of miR-155 (Fig. 3d), proving that the modifications of the target site of KRAS 3' UTR are able to block the function of this miRNA.

In agreement with the capacity of miR-155 to target KRAS mRNA, the relative protein amounts are higher in $c b x 7$ KO MEFs in comparison with the WT (Fig. 3e).

According to the results obtained on $c b x 7$ null MEFs, the levels of KRAS protein were found downregulated in A549 cells (human lung carcinoma line), overexpressing the CBX7 protein (Fig. 4a), in which we found higher miR-155 levels with respect to the same cells transfected with the control vector (Fig. 4b).

\section{miR-155 expression positively correlates with $C B X 7$ in human colon carcinoma}

Then, we have evaluated miR-155 and the $C B X 7$ gene expression in colon carcinoma tissues by qRT-PCR. As presented in Fig. 5, downregulation of both miR-155 and $C B X 7$ was observed in most of the carcinoma samples in comparison with the normal colon mucosa. Linear regression between $C B X 7$ and miR-155 expression is also reported (Fig. 5).

The direct correlation between $C B X 7$ and miR-155 expression suggests that these genes are co-regulated in human colon carcinomas. The presence of a mutated KRAs status does seem to have any correlation with miR-155 or $C B X 7$ expression.

We have also analyzed KRAS expression at mRNA level. These results are shown in the Additional file 1: Figure S1. There is no significant correlation with $C B X 7$ and miR-155 levels. It is likely that several other factors act on KRAS mRNA levels in vivo, and maybe the effect of miR-155 is exerted particularly at translation level.

\section{Discussion}

$C B X 7$ has been already proposed as tumor suppressor gene, since several studies have demonstrated that its expression is drastically downregulated in most of the malignant neoplasias [4], and the development of liver and lung carcinomas in $C b x-7$ null mice further supports the CBX7 tumor suppressor activity [8]. Moreover, the restoration of $C B X 7$ expression in carcinoma cells of different origin results into a reduced growth rate $[5,7,11$, 33], blocking the cells in the G1 phase of the cell cycle. Very recently, we have also reported that reestablishment of $C B X 7$ expression in two cell lines of human lung carcinoma, in which it was undetectable, yields a diminished proliferation and an improved apoptosis after drug exposure [21]. However, CBX7 has been reported to exert also oncogenic activity. Indeed, it was shown that $C b x 7$ expression in mice lymphoid compartment

Table 2 List of the most studied and down-regulated miRNAs in WT MEFs (cbx $7^{+/+}$) vs KO MEFs (cbx $7^{-/}$)

\begin{tabular}{llrrr}
\hline Unique Id & Geom. Mean of intensities: $\left(\mathrm{cbx} 7^{+/+}\right)$ & Geom. Mean of intensities: $\left(\mathrm{cbx} 7^{-/}\right)$ & $\begin{array}{l}\text { Ratio of geom } \\
\text { Means } \\
\left(\mathrm{cbx7} 7^{+/+} \text {vs cbx7 } 7^{-/-}\right)\end{array}$ & $\begin{array}{l}\text { Parametric } \\
p \text {-value }\end{array}$ \\
\hline Mmu-mir-137 & 113.9 & 687.8 & 0.166 & 0.0092591 \\
Mmu-mir-181a-5p & 1124.6 & 2092.2 & 0.538 & 0.0030335 \\
Mmu-miR-719 & 18 & 186.1 & 0.097 & 0.0002231 \\
Mmu-mir-199-5p & 445.9 & 1481.1 & 0.301 & 0.0001483 \\
\hline
\end{tabular}




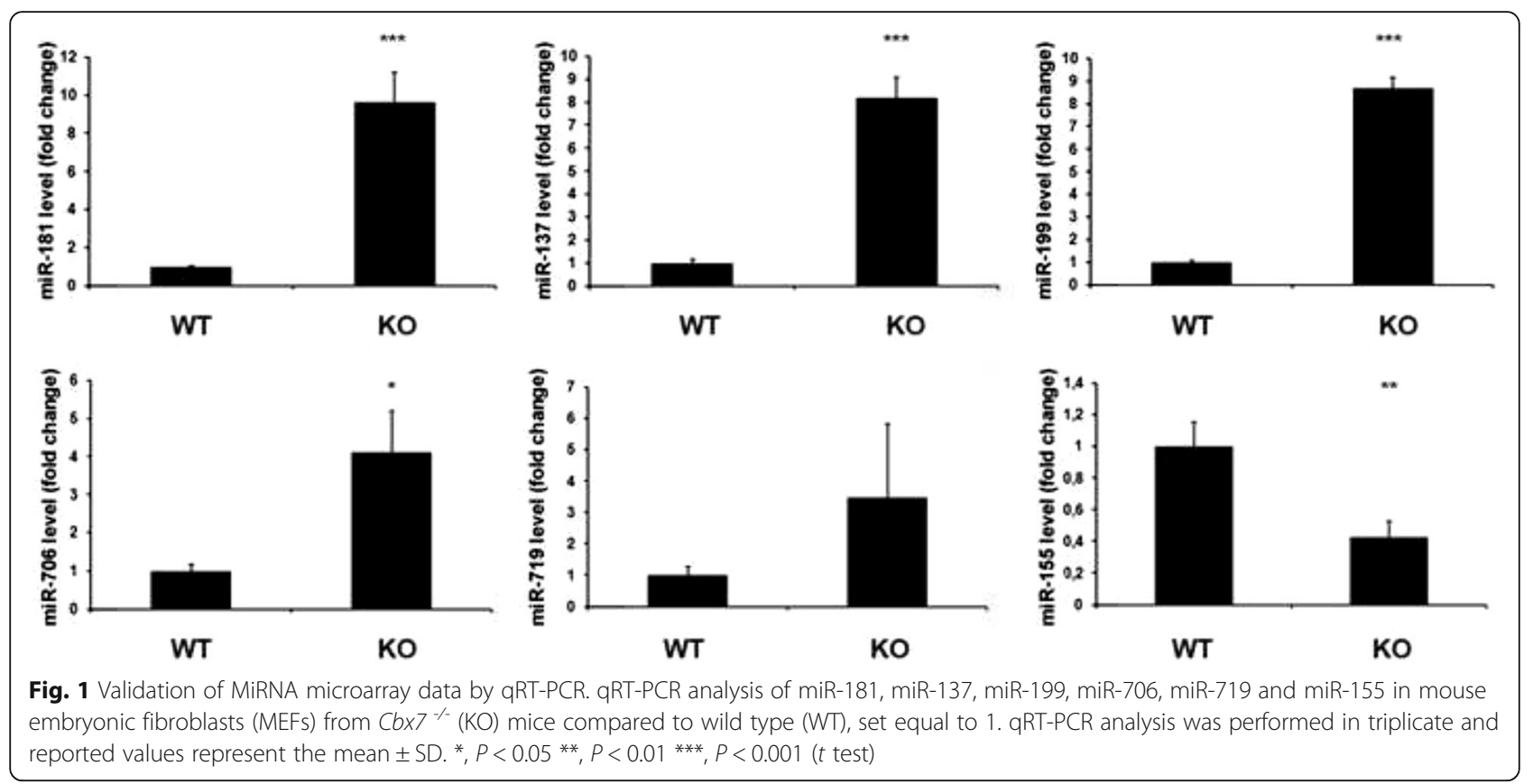

can promote $\mathrm{T}$ cell lymphomagenesis and, working together with c-Myc, produces aggressive B cell lymphomas by downregulating Ink4a/Arf locus [1]. Equally, CBX7-positive patients affected by ovarian carcinoma showed significantly shorter overall and progression-free survival rates than those of the CBX7-negative patients. Moreover, $C B X 7$ knockdown significantly reduced cell viability in two ovarian carcinoma cell lines compared to the control cells, likely by the upregulation of tumor necrosis factor-related apoptosis-inducing ligand (TRAIL) [34]. Recently, we have reported that CBX7 regulates several genes involved in tumor progression [12, 35] such as E cadherin, cyclin E, SPP1 likely accounting for the critical role of $\mathrm{CBX} 7$ in carcinogenesis.

In this study, we have envisaged the hypothesis that CBX7 tumor suppressor or oncogenic role may be also mediated by the regulation of miRNA expression since the role of miRNAs in cancer development and progression has been frequently reported [36]. Consistently, we previously demonstrated that $\mathrm{CBX} 7$ negatively regulates the expression of miR-181 that has among its targets CBX7, creating a synergistic loop that contributes to breast cancer progression [11].

Then, we have studied the miRNA expression profile of $C b x 7$ null MEFs versus the wt. Nine miRNAs were found downregulated and twenty upregulated with a fold change higher than 2 in the $C b x 7$ KO MEFs with respect to WT counterparts. We first validated the results of the array analyzing, by qRT-PCR, 5 deregulated miRNAs in $C b x 7 \mathrm{KO}$ and WT MEFs.

On the basis of the opposite functions of CBX7, as oncogene and oncosuppressor, it was not surprising to find that CBX7 was able to regulate in opposite sense miRNAs that have recognized to have oncogenic functions, such as miR-
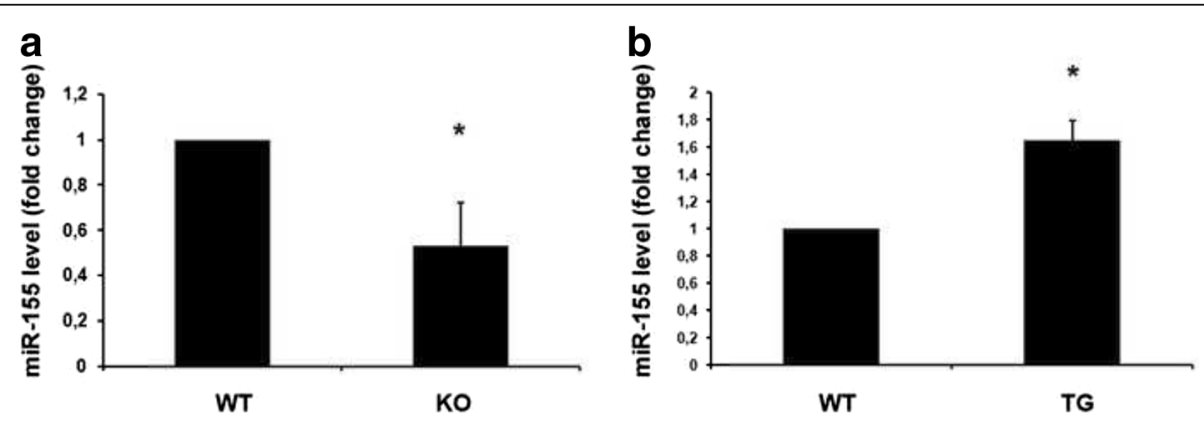

Fig. 2 MiR-155 espression in MEFs derived from Cbx7 null and Cbx7 transgenic mice. a qRT-PCR analysis of miR-155 in MEFs derived from three Cbx7 knock out lines (KO1, KO2, KO3) and control WT mice and $\mathbf{b}$ in MEFs derived from Cbx7 transgenic lines (TG1, TG2, TG3). qRT-PCR analyses were performed in triplicate and reported values represent the mean \pm SD. ${ }^{*}, P<0.05$ ( $t$ test) 

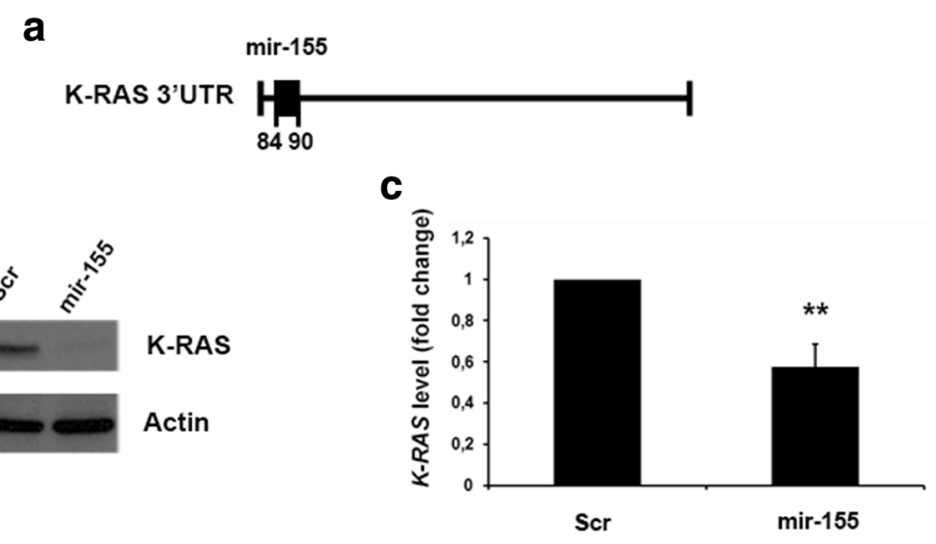

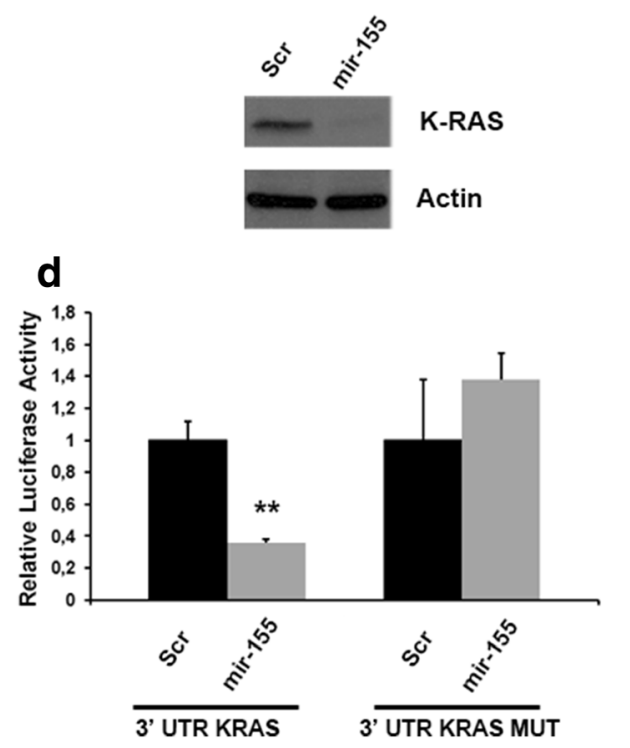

b

e

WT KO

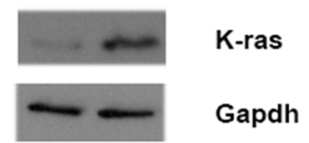

Fig. 3 MiR-155 targets KRAS. a Schematic representation of KRAS 3' UTR and relative position of the predicted miRNA- binding site. b Western blot analysis of KRAS protein levels in HEK-293 cells transfected with miR-155 or a scrambled oligonucleotide. Actin expression was analyzed as loading control. A representative experiment is shown. c qRT-PCR analyses of KRAS mRNA expression in HEK-293 cells transfected with miR-155 or a scrambled oligonucleotide, normalized with G6PD. qRT-PCR analysis was performed in triplicate and reported values represent the mean \pm SD. d Relative luciferase activity in HEK-293 cells transiently transfected with 3'UTR-KRAS and 3'UTR-KRAS mutated in the miR-155 seed sequence along with the miR 155 or with a scrambled oligonuclotide. e Western blot analysis of KRAS protein in MEFs Cbx $7^{+/+}(\mathrm{WT})$ and $\mathrm{Cbx} 7^{-/-}(\mathrm{KO})$. Gapdh expression was analyzed as loading control. **, $P<0.01$ ( $t$ test)

a
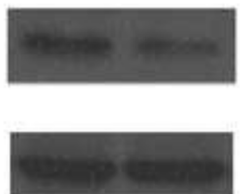

K-RAS

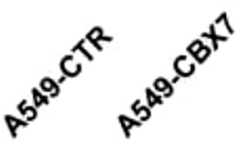

b

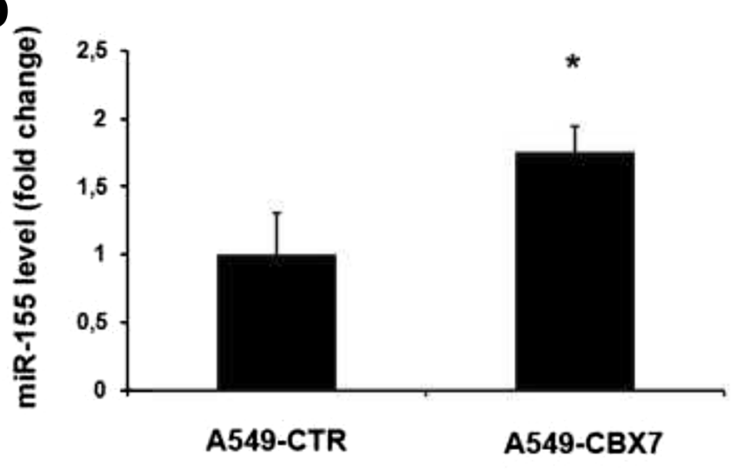

Fig. 4 KRAS and miR-155 espression in A549 cell clones transfected with CBX7. aWestern blot analysis of KRAS protein in A549 cells expressing CBX7 (A549-CBX7) versus A549 cells transfected with the empty vector (A549-CTR). Actin expression was analyzed as loading control. b qRT-PCR analysis of miR-155 expression in A549-CBX7 cells versus A549-CTR cells, normalized with G6PD. QRT-PCR analysis was performed in triplicate and reported values represent the mean $\pm \mathrm{SD}$. ${ }^{*}, P<0.05$ ( $t$ test) 


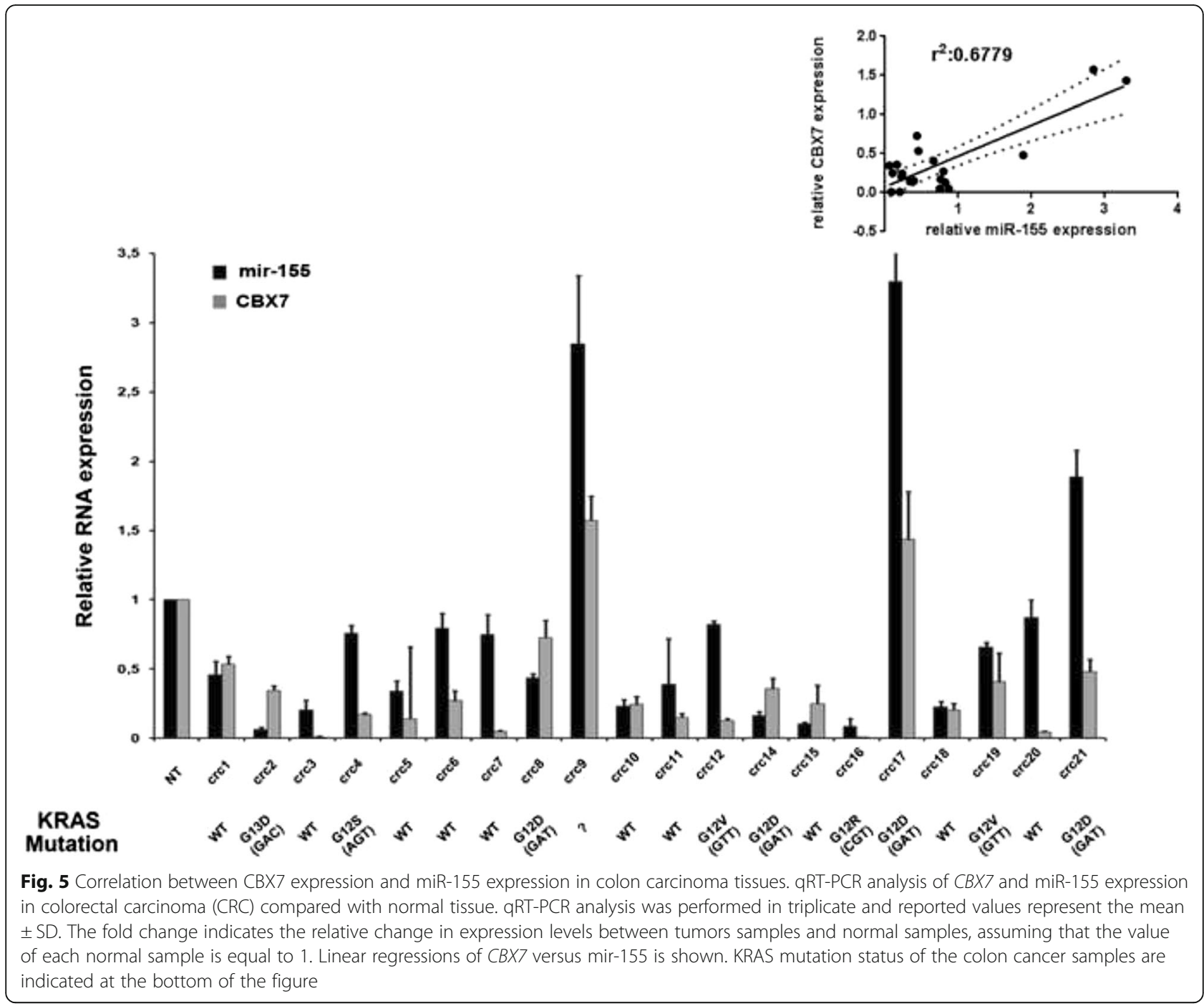

199 (negatively regulated) and miR-155, miR-221 and miR222 (positively regulated). Equally, we observe that a potential oncosuppressor gene, such as miR-137, is regulated negatively, whereas another one, such as miR-323, is regulated positively. It is also not unlikely that the modulation of these miRNAs might be specific of MEFs and that CBX7 could regulate the same miRNA positively or negatively depending on the cellular context. Therefore, we retain that the recent results evidencing a role of the loss of CBX7 expression in the progression of human colon cancer might be also explained by the ability of $\mathrm{CBX} 7$ to modulate the expression of several miRNAs.

Subsequently, we decided to focus on the miR-155 since it was one of the most downregulated miRNAs in Cbx7 KO MEFs. Moreover, miR-155 is the most commonly miRNA overexpressed in malignancies among the cancer-related miRNAs [28]. Bcl6, HDAC4, msh2, msh6, mlh1 have been already identified as targets of miR-155 [26]. In liposarcoma casein kinase $1-\alpha \quad(\mathrm{CK} 1-\alpha)$ is targeted by miR-155, enhancing beta-catenin and cyclin D1 [27]. In this study we have identified KRAS as target of miR-155, since overexpression of miR-155 leads to a drastic reduction of the KRAS mRNA and protein levels indicating an effect of miR-155 also on KRAS mRNA degradation. Consistently, higher KRAS protein levels were detected in $C b x 7$ null MEFs in comparison with the WT. The same result was achieved when $C B X 7$ expression was restored in lung carcinoma cells. However, the analysis of KRAS specific mRNA does not reveal any correlation with miR-155 and CBX7 expression. Maybe this correlation is present at protein levels, and we can also hypothesize that the regulation of KRAS by miR-155 is specific of some tissues depending on the cellular context.

It is likely that the targeting of KRAS may have a role in the anti-apoptotic activity of miR-155 observed in monocytic differentiation where miR-155 seems to have as targets other anti-apoptotic factors such as RPS6KA3, 
SGK3, and RHEB. It is likely that this anti-apoptotic activity may be important for the growth of MEFs, also associated to the increased mR-221 and miR-222 levels that are able to target p27 [37], a critical negative regulator of the cell cycle.

Interestingly, CBX7 seems to positively regulate miR323: this can account for the reduced miR-323 expression in prostate cancer where CBX7 is drastically downregulated [38] and miR-323 has a tumor suppressor activity by targeting AdipoRI [39].

\section{Conclusion}

In conclusion, these studies demonstrate that $\mathrm{CBX7}$ is able to positively regulate miR-155 and identify KRAS as one of the target genes of this miRNA likely accounting for the anti-apoptotic activity ascribed to miR-155 in some tissue context.

\section{Additional file}

Additional file 1: Figure S1. qRT-PCR analysis of KRAS expression in colorectal carcinoma (CRC) compared with normal tissue. qRT-PCR analysis was performed in triplicate and reported values represent the mean \pm SD. The fold change indicates the relative change in expression levels between tumors samples and normal samples, assuming that the value of each normal sample is equal to 1. (TIF $757 \mathrm{~kb}$ )

\section{Abbreviations}

HEK: Human embryonic kidney 293 cells; KO: Knockout; MEF: Mouse embryonic fibroblasts; miRNA: microRNA; PRC: Polycomb repressive complex: qRT-PCR: Quantitative real time PCR; TG: Transgenic; WT: Wild-type

\section{Acknowledgements}

Dr Pierlorenzo Pallante for his helpful supports.

\section{Funding}

This study has been supported by grants from: PRIN-MIUR (2011-2014): Basi molecolari dei processi di carcinogenesi polmonare: caratterizzazione del network trascrizionale e di microRNA a valle delle vie di trasduzione del segnale attive durante lo sviluppo embrionale in cellule staminali tumorali; PNR-CNR Aging Program 2012-2014, POR Campania FSE 2007-2013 (CREMe), CNR Flagship Projects (Epigenomics-EPIGEN, PON 01-02782 Nuove strategie nanotecnologiche per la messa a punto di farmaci e presidi diagnostici diretti verso cellule cancerose circolanti), Associazione Italiana per la Ricerca sul Cancro (AIRC IG 11477).

Availability of data and materials

Data is available in the Additional file 1.

\section{Authors' contribution}

FF and AF conceived and designed the study. FF with MDM, FE, RS, and SP performed all experiments. UM and GP collected colorectal samples. FF, FE, $\mathrm{CA}$ and $\mathrm{AF}$ analyzed most data. FF, FE and AF wrote the manuscript with contributions from all authors. All authors read and approved the final manuscript.

\section{Competing interests}

The authors declare that they have no competing interests.

\section{Ethics approval and consent to participate}

The ethics committees of the University of Naples "Federico II" and Second University of Naples allowed the execution of these investigations. Written informed consent was obtained from all of the patients included in this study

\section{Author details}

"Istituto di Endocrinologia ed Oncologia Sperimentale "G. Salvatore" - CNR c/o Dipartimento di Medicina Molecolare e Biotecnologie Mediche, Università degli Studi di Napoli "Federico II", Naples, Italy. ${ }^{2}$ Dipartimento di Sanità Pubblica, Università degli Studi di Napoli "Federico II", Naples, Italy. ${ }^{3}$ Unità di Chirurgia Colorettale, Dipartimento di Scienze Mediche, Chirurgiche, Neurologiche e dell'Invecchiamento, Seconda Università di Napoli, Naples, Italy. IIstituto Nazionale dei Tumori, Fondazione Pascale, Naples, Italy.

Received: 16 April 2016 Accepted: 24 February 2017

Published online: 04 March 2017

\section{References}

1. Scott CL, Gil J, Hernando E, Teruya-Feldstein J, Narita M, Martinez D, et al. Role of the chromobox protein CBX7 in lymphomagenesis. Proc Natl Acad Sci U S A. 2007;104:5389-94.

2. Schuettengruber B, Chourrout D, Vervoort M, Leblanc B, Cavalli G. Genome regulation by polycomb and trithorax proteins. Cell. 2007;128:735-45.

3. Wu JL, Lessard J, Crabtree GR. Understanding the words of chromatin regulation. Cell. 2009;136:200-6.

4. Pallante P, Forzati F, Federico A, Arra C, Fusco A. Polycomb protein family member CBX7 plays a critical role in cancer progression. Am J Cancer Res. 2015;5:1594-601.

5. Pallante P, Federico A, Berlingieri MT, Bianco M, Ferraro A, Forzati F, et al. Loss of the CBX7 gene expression correlates with a highly malignant phenotype in thyroid cancer. Cancer Res. 2008;68:6770-8,

6. Karamitopoulou E, Pallante P, Zlobec I, Tornillo L, Carafa V, Schaffner T, et al. Eur J Cancer. 2010:46:1438-44.

7. Pallante P, Terracciano L, Carafa V, Schneider S, Zlobec I, Lugli A, et al. The loss of the CBX7 gene expression represents an adverse prognostic marker for survival of colon carcinoma patients. Eur J Cancer. 2010:46:2304-13.

8. Forzati F, Federico A, Pallante P, Abbate A, Esposito F, Malapelle U, et al. CBX7 is a tumor suppressor in mice and humans. J Clin Invest. 2012;122:612-23.

9. Jiang Z, Guo J, Xiao B, Miao Y, Huang R, Li D, et al. Increased expression of miR-421 in human gastric carcinoma and its clinical association. J Gastroenterol. 2010;45:17-23.

10. Hinz S, Kempkensteffen C, Christoph F, Krause H, Schrader M, Schostak M, et al. Expression parameters of the polycomb group proteins BMI1, SUZ12, RING1 and CBX7 in urothelial carcinoma of the bladder and their prognostic relevance. Tumor Biol. 2008;29:323-29.

11. Mansueto G, Forzati F, Ferraro A, Pallante P, Bianco M, Esposito F, et al. Identification of a new pathway for tumor progression: microRNA-181b upregulation and CBX7 down-regulation by HMGA1 protein. Genes Cancer. 2010;1:210-24.

12. Federico A, Pallante $\mathrm{P}$, Bianco M, Ferraro A, Esposito F, Monti M, et al. Chromobox protein homologue 7 protein, with decreased expression in human carcinomas, positively regulates $\mathrm{E}$-cadherin expression by interacting with the histone deacetylase 2 protein. Cancer Res. 2009:69:7079-87.

13. Thiery JP. Epithelial-mesenchymal transitions in tumor progression. Nat Rev Cancer. 2002;2:442-54

14. Thiery JP, Sleeman J. Complex networks orchestrate epithelial-mesenchymal transitions. Nat Rev Mol Cell Biol. 2006;7:131-42.

15. Sepe R, Formisano U, Federico A, Forzati F, Bastos AU, D'Angelo D, et al. CBX7 and HMGA1b proteins act in opposite way on the regulation of the SPP1 gene expression. Oncotarget. 2015;6:2680-92.

16. Ambros V. The functions of animal microRNAs. Nature. 2004;431:350-5.

17. Bartel DP. MicroRNAs: genomics, biogenesis, mechanism, and function. Cell. 2004;116:281-97.

18. Lewis $B P$, Burge $C B$, Bartel DP. Conserved seed pairing, often flanked by adenosines, indicates that thousands of human genes are microRNA targets. Cell. 2005;120:15-20.

19. Bartel DP. MicroRNAs: target recognition and regulatory functions. Cell. 2009;136:215-33.

20. Hwang HW, Mendell JT. MicroRNAs in cell proliferation, cell death, and tumorigenesis. Br J Cancer. 2007;96:40-4.

21. Cacciola NA, Sepe R, Forzati F, Federico A, Pellecchia S, Malapelle U, et al. Restoration of CBX7 expression increases the susceptibility of human lung carcinoma cells to irinotecan treatment. Naunyn Schmiedebergs Arch Pharmacol. 2015;388:1179-86. 
22. Liu CG, Calin GA, Meloon B, Gamliel N, Sevignani C, Ferracin M, et al. An oligonucleotide microchip for genome-wide microRNA profiling in human and mouse tissues. Proc Natl Acad Sci U S A. 2004;101:9740-4.

23. Mussnich P, D'Angelo D, Leone V, Croce CM, Fusco A. The High Mobility Group A proteins contribute to thyroid cell transformation by regulating miR-603 and miR-10b expression. Mol Oncol. 2013;7:531-42.

24. Leone V, Langella C, Esposito F, De Martino M, Decaussin-Petrucci M, Chiappetta G, et al. miR-130b-3p upregulation contributes to the development of thyroid adenomas Targeting CCDC6 gene. Eur Thyroid J. 2015;4:213-21.

25. Martinez Hoyos J, Ferraro A, Sacchetti S, Keller S, De Martino I, Borbone E, et al. HAND1 gene expression is negatively regulated by the High Mobility Group A1 proteins and is drastically reduced in human thyroid carcinomas. Oncogene. 2009;28:876-85.

26. Higgs $G$, Slack F. The multiple roles of microRNA-155 in oncogenesis. J Clin Bioinforma. 2013;3:17.

27. Zhang P, Bill K, Liu J, Young E, Peng T, Bolshakov $S$, et al. MiR-155 is a liposarcoma oncogene that targets casein kinase-1a and enhances $\beta$ catenin signaling. Cancer Res. 2012;72:1751-62.

28. Sandhu SK, Volinia S, Costinean S, Galasso M, Neinast R, Santhanam R, et al. miR-155 targets histone deacetylase 4 (HDAC4) and impairs transcriptional activity of B-cell lymphoma 6 (BCL6) in the E $\mu$-miR-155 transgenic mouse model. Proc Natl Acad Sci U S A. 2012;109:20047-52.

29. Karachaliou N, Mayo C, Costa C, Magrí I, Gimenez-Capitan A, Molina-Vila MA, et al. KRAS mutations in lung cancer. Clin Lung Cancer. 2013;14:205-14.

30. Wood LD, Hruban RH. Pathology and molecular genetics of pancreatic neoplasms. Cancer J. 2012;18:492-501.

31. Arrington AK, Heinrich EL, Lee W, Duldulao M, Patel S, Sanchez J, et al, Prognostic and predictive roles of KRAS mutation in colorectal cancer. Int J Mol Sci. 2012;13:12153-68.

32. Avgoustou C, Giannousis D, Penlidis P, Gigondi J, Boukis C, Chatziioannou A. Immunohistochemical analysis of K-RAS expression in curatively treated colorectal cancer patients: Correlations of clinicopathological features with clinical outcome. Hellenic J Surg. 2013;85:165-74.

33. Li Q, Wang X, Lu Z, Zhang B, Guan Z, Liu Z, et al. Polycomb CBX7 directly controls trimethylation of histone $\mathrm{H} 3$ at lysine 9 at the p16 locus. PLoS One. 2010:5:e13732

34. Shinjo K, Yamashita Y, Yamamoto E, Akatsuka S, Uno N, Kamiya A, et al. Int Expression of chromobox homolog 7 (CBX7) is associated with poor prognosis in ovarian clear cell adenocarcinoma via TRAIL-induced apoptotic pathway regulation. J Cancer. 2014;135:308-18.

35. Pallante P, Sepe R, Federico A, Forzati F, Bianco M. Fusco A CBX7 modulates the expression of genes critical for cancer progression. PLoS One. 2014;9: e98295.

36. Calin GA, Croce CM. MicroRNA signatures in human cancers. Nat Rev Cancer. 2006:6:857-66.

37. Visone R, Russo L, Pallante P, De Martino I, Ferraro A, Leone V, et al. MicroRNAs (miR)-221 and miR-222, both overexpressed in human thyroid papillary carcinomas, regulate p27Kip1 protein levels and cell cycle. Endocr Relat Cancer. 2007:14:791-8.

38. Gene Expression Omnibus. http://www.ncbinlm.nih.gov/geo/query/acc. cgi?acc $=$ GSE21032

39. Gao Q, Yao X, Zheng J. MiR-323 inhibits prostate cancer vascularization through adiponectin receptor. Cell Physiol Biochem. 2015;36:1491-8.

\section{Submit your next manuscript to BioMed Central and we will help you at every step:}

- We accept pre-submission inquiries

- Our selector tool helps you to find the most relevant journal

- We provide round the clock customer support

- Convenient online submission

- Thorough peer review

- Inclusion in PubMed and all major indexing services

- Maximum visibility for your research

Submit your manuscript at www.biomedcentral.com/submit

C Biomed Central 